ИЗВЕСТИЯ АКАДЕМИИ НАУК ЭСТОНСКОП ССР. ТОМ 27 ФИЗНКА * МАТЕМАТИКА, 1978, № 4

\title{
О КЛАССИФИКАЦИИ РЕЗОНАНСНОГО ВТОРИЧНОГО СВЕЧЕНИЯ ЭКСИТОНОВ
}

\author{
(Представлена В. Хижняковым)
}

О проблеме классификации резонансного вторичного свечения (РВС) экситонов высказывается немало противоречивых суждений. Так, в ['] утверждается, что в случае экситонов горячая люминесценция (ГЛ) и резонансное комбинационное рассеяние (PKР) неразличимы. В $\left[{ }^{2}\right]$, хотя и признается различие между этими двумя компонентами РВС, однако для их разделения используются неприемлемые для этой цели критерии (температурная зависимость спектра и сравнение интенсивностей двух- и трехфононных спектров (см. $\left.\left.\left[{ }^{3}\right]\right)\right)$. В $\left[{ }^{3}\right]$ была проведена классификация спектров РВС экситонов с рождением двух и трех оптических фононов на основе спектрального критерия: в случае ГЛ (как и в случае обычной люминесценции (ОЛ)) вероятность двухфотонного процесса может быть представлена в виде произведения вероятностей поглощения первичного фотона и излучения вторичного. В случае РКР такое представление невозможно.

Важность отмеченной проблемы обусловлена тем, что РКР и ГЛ два качественно различных компонента РВС [ $\left.{ }^{4}\right]$.

Проблема классификации РВС экситонов является частным случаем общей проблемы классификации РВС вещества, так что основные принципы должны быть общими. Представляет поэтому интерес сравнить выводы $\left[{ }^{3}\right]$ с результатами, следующими из временного критерия, который использовался в $\left[{ }^{5,6}\right]$ для классификации РВС примесных центров кристаллов, где различия между РКР и ГЛ могут проявляться очень четко. Согласно этому критерию, излучение, возникающее до затухания фазовой памяти в системе, есть рассеяние; излучение, происходящее после фазовой релаксации, но в ходе энергетической, есть ГЛ; излучение после обоих типов релаксации - ОЛ.

В данной работе этот критерий используется для классификации спектра РВС экситонов Френкеля с рождением двух оптических фононов при нулевой температуре. При этом показывается, что спектральный критерий $\left[{ }^{3}\right]$ полностью согласуется с временным $\left[{ }^{5,6}\right]$.

Воспользуемся моделью кристалла из $\left[{ }^{3}\right]$ : в идеальном кристалле имеется зона экситонов Френкеля, хорошо отделенная от других состояний кристалла. Будем предполагать, что экситоны взаимодействуют с одной ветвыю оптических фононов, так что гамильтониан кристалла имеет вид 


$$
\begin{aligned}
& H_{s}=H_{e}+H_{L}+H_{e L}, \quad H_{e}=\sum_{\vec{k}} \varepsilon(\vec{k}) \underset{\vec{k}}{a_{\vec{k}}^{+}} a_{\vec{k}},
\end{aligned}
$$

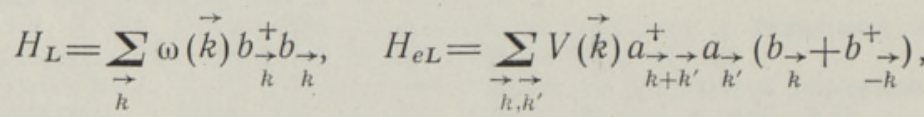

где $\varepsilon(\vec{k}), \omega(\vec{k})$ - энергии экситонов и фононов с волновым вектором $\vec{k}$ (выбрана система единиц, в которой $\bar{\hbar}=1$ ); $\underset{\vec{k}}{a} \underset{\vec{k}}{+}, \underset{k}{a_{\rightarrow}}, \underset{k}{+} b_{\vec{k}}-$ операторы рождения и уничтожения экситонов и фононов с волновым вектором $\vec{k} ; V(\vec{k})$ - константы экситон-фононного взаимодействия, зависящие, по предположению, лишь от волнового вектора фонона.

Предполагается, что поляритонный эффект не существен. В кристалле распространяется фотон поляризации $\lambda_{1}$ и частоты $\Omega_{1}$, лежащей в интервале

$$
\varepsilon_{0}+2 \omega_{0}<\Omega_{1}<\varepsilon_{0}+B
$$

где $\varepsilon_{0}$ - энергия дна экситонной зоны, $\omega_{0}$ - предельная частота оптического фонона, $B$ - ширина экситонной зоны. Условие (1) означает, что рассматриваемый спектр не может относиться к ОЛ. При сделанных предположениях вероятность излучения вторичного фотона частоты $\Omega_{2}$ и поляризащии $\lambda_{2}$ получается из формулы теории возмущений второго порядка по взаимодействию света с кристаллом. Используя интегральные представления для $\delta$-функции и резольвенты, представим эту формулу в виде

$$
W=\left|T_{\overrightarrow{x_{1} \lambda_{1}}} T_{\vec{x}_{2} \lambda_{2}}\right|^{2} \int_{-\infty}^{\infty} d t \iint_{0}^{\infty} d \tau_{1} d \tau_{2} \mathrm{e}^{i\left(\Omega_{1}-\Omega_{2}\right) t} \mathrm{e}^{i \Omega_{1}\left(\tau_{2}-\tau_{1}\right)} A\left(t, \tau_{1}, \tau_{2}\right),
$$

где, для рассматриваемого спектра с рождением двух фононов при нулевой температуре (см. $\left.\left[{ }^{3}\right]\right)$,

$$
\begin{aligned}
& A\left(t, \tau_{1}, \tau_{2}\right)=A_{1}\left(t, \tau_{1}, \tau_{2}\right)+A_{2}\left(t, \tau_{1}, \tau_{2}\right), \\
& A_{j}=\mathrm{e}^{i \varepsilon\left(\boldsymbol{x}_{1}\right)\left(\tau_{1}-\tau_{2}\right)} \underset{\overrightarrow{\vec{k}}}{\underset{\vec{k}}{\Sigma}}\left|V(\vec{k}) V\left(\overrightarrow{\varkappa_{1}}-\vec{k}-\overrightarrow{\varkappa_{2}}\right)\right|^{2} \mathrm{e}^{-i t\left[\omega(\vec{k})+\omega\left(\hat{x}_{1}-\vec{k}-\vec{x}_{2}\right)\right]} g_{2}\left(\tau_{1}, \vec{k}\right) g_{j}^{*}\left(\tau_{2}, \vec{k}\right), \\
& g_{j}(\tau, \vec{k})=\frac{1}{\varphi(\vec{k})-\varphi_{j}(\vec{k})}\left\{\frac{\mathrm{e}^{i \varphi(\vec{k}) \tau}-1}{\varphi(\vec{k})}-\frac{\mathrm{e}^{i \varphi_{j}(\vec{k}) \tau}-1}{\varphi_{j}(\vec{k})}\right\}, \\
& \varphi_{1}(\vec{k})=\varepsilon^{\prime}\left(\overrightarrow{\varkappa_{2}}+\vec{k}\right)+\omega\left(\vec{\varkappa}_{1}-\vec{k}-\vec{x}_{2}\right)-\varepsilon\left(\vec{\varkappa}_{1}\right) \text {, } \\
& \varphi_{2}(\vec{k})=\varepsilon^{\prime}\left(\overrightarrow{\varkappa_{1}}-\vec{k}\right)+\omega(\vec{k})-\varepsilon\left(\overrightarrow{\varkappa_{1}}\right), \\
& \varphi(\vec{k})=\varepsilon\left(\vec{x}_{2}\right)+\omega\left(\overrightarrow{x_{1}}-\vec{k}-\vec{x}_{2}\right)+\omega(\vec{k})-\varepsilon\left(\vec{x}_{1}\right) \text {. }
\end{aligned}
$$

В формулах (2) и (3) учтено, что гамильтониан экситон-фотонного взаимодействия в данном случае имеет вид

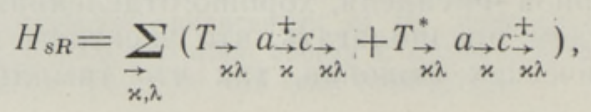




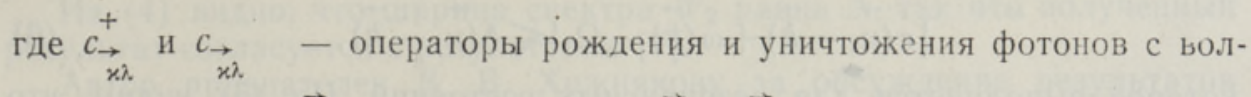
новым вектором $\vec{x}$ и поляризацией $\lambda, \vec{x}_{1}$ и $\vec{x}_{2}$ - волновые векторы первичного и вторичного фотонов. Использован наинизший (второй) порядок теории возмущений по оператору экситон-фононного взаимодействия; более высокие порядки учтены путем введения распадной ширины экситона: $\varepsilon^{\prime}(\vec{k})=\varepsilon(\vec{k})+i \delta(\vec{k})$.

Из (2) и (3) получаем

$$
\begin{gathered}
W=W_{1}+W_{2}, \\
W_{1}=\frac{2 \pi\left|T_{\hat{x}_{1} \lambda_{1}} T_{\vec{x}_{2} \lambda_{2}}\right|^{2}}{\left[\varepsilon\left(\overrightarrow{x_{1}}\right)-\Omega_{1}\right]^{2}\left[\varepsilon\left(\overrightarrow{x_{2}}\right)-\Omega_{2}\right]^{2}} \times
\end{gathered}
$$

$\times \underset{\vec{k}}{\sum} \frac{\left.\left|V(\vec{k}) V\left(\vec{x}_{1}-\vec{k}-\overrightarrow{x_{2}}\right)\right|^{2} \delta\left(\Omega_{1}-\Omega_{2}-\omega(\vec{k})-\omega\left(\overrightarrow{x_{1}}-\vec{k}-\overrightarrow{x_{2}}\right)\right)+\omega(\vec{k})-\Omega_{1}\right]\left[\varepsilon^{\prime *}\left(\overrightarrow{x_{2}}+\vec{k}\right)+\omega\left(\overrightarrow{x_{1}}-\vec{k}-\overrightarrow{x_{2}}\right)-\Omega_{1}\right]}{,}$

$$
W_{2}=\frac{2 \pi\left|T_{\vec{x}_{1} \lambda_{1}} T_{\vec{\kappa}_{2} \lambda_{2}}\right|^{2}}{\left[\varepsilon\left(\overrightarrow{\varkappa_{1}}\right)-\Omega_{1}\right]^{2}\left[\varepsilon\left(\overrightarrow{\varkappa_{2}}\right)-\Omega_{2}\right]^{2}} \times
$$

$\times \underset{\vec{k}}{\sum} \frac{\left|V(\vec{k}) V\left(\vec{x}_{1}-\vec{k}-\overrightarrow{x_{2}}\right)\right|^{2} \delta\left(\Omega_{1}-\Omega_{2}-\omega(\vec{k})-\omega\left(\overrightarrow{x_{1}}-\vec{k}-\overrightarrow{x_{2}}\right)\right)}{\left[\varepsilon\left(\overrightarrow{x_{1}}-\vec{k}\right)+\omega(\vec{k})-\Omega_{1}\right]^{2}+\delta^{2}\left(\overrightarrow{x_{1}}-\vec{k}\right)}$.

$W_{1}$ - слагаемое интерференционного типа. Как показано в $\left[{ }^{7}\right]$, относительные величины $W_{1} \cdot$ и $W_{2}$ могут существенно зависеть от взаимного расположения векторов $\vec{\varkappa}_{1}$ и $\vec{\varkappa}_{2}$. При изменении их относительного расположения может меняться и вид зависимости $W_{1}\left(\Omega_{1}, \Omega_{2}\right)$. Как видно из (4) (см. также $\left.\left[{ }^{7}\right]\right)$, в этом случае $W_{1}\left(\Omega_{\mathfrak{\digamma}}, \Omega_{2}\right) \approx W_{2_{2}}\left(\Omega_{1}, \Omega_{2}\right)$ при «рассеянии» назад $\vec{x}_{1} \approx-\vec{x}_{2}$ и, вообще говоря, $W_{1} \neq W_{2}$ для других направлений $\vec{x}_{2}$. Ясно, что при $W_{1}\left(\Omega_{1}, \Omega_{2}\right) \approx W_{2}\left(\Omega_{1}, \Omega_{2}\right)$ слагаемое $W_{1}$ следует классифицировать так же, как $W_{2}$. При $W_{1} \neq W_{2}$ излучение, описываемое $W_{1}$, классифицируется как интерференционное.

Рассмотрим излучение, описываемое слагаемым $W_{2}$. Легко убедиться, что при условии (1) основной вклад в спектр дает следующая часть коррелятора $A_{2}\left(t, \tau_{1}, \tau_{2}\right)$ :

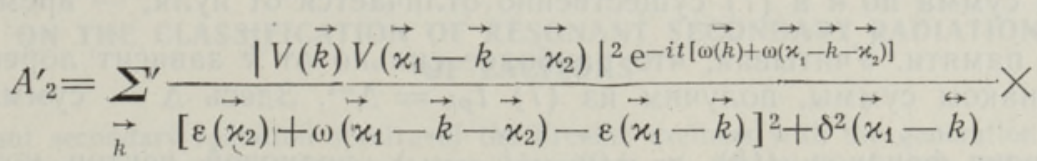

$$
\begin{aligned}
& \times \frac{\mathrm{e}^{i\left(\tau_{1}-\tau_{2}\right)\left[\varepsilon\left(\overrightarrow{x_{1}}-\vec{k}\right)+\omega(\vec{k})\right]} \mathrm{e}^{-\left(\tau_{1}+\tau_{2}\right) \delta\left(\vec{x}_{1}-\vec{k}\right)}}{\left[\varepsilon\left(\overrightarrow{\varkappa_{1}}-\vec{k}\right)+\omega(\vec{k})-\varepsilon\left(\vec{\varkappa}_{1}\right)\right]^{2}+\delta^{2}\left(\overrightarrow{\varkappa_{1}}-\vec{k}\right)},
\end{aligned}
$$

где штрих у знака суммы обозначает, что суммнрование проводится по волновым векторам, удовлетворяющим условию 


$$
\left|\varepsilon\left(\vec{x}_{1}-\vec{k}\right)+\omega(\vec{k})-\Omega_{1}\right| \leqslant \delta\left(\vec{x}_{1}-\vec{k}\right),
$$

которое выполняется для резонансных состояний. Из (5) видим, что $\left(\tau_{1}+\tau_{2}\right) / 2 \leqslant(2 \delta)^{-1}$, где $\delta-$ средняя распадная ширина экситонов, удовлетворяющих условию (6). Соответствующий процесс преобразования фотонов имеет вид:

$$
\begin{aligned}
& \left|\Omega_{1}\right\rangle|0\rangle|0\rangle \rightarrow|0\rangle\left|\varepsilon\left(\overrightarrow{x_{1}}\right)\right\rangle|0\rangle \rightarrow|0\rangle\left|\varepsilon\left(\vec{x}_{1}-\vec{k}\right)\right\rangle|\omega(\vec{k})\rangle \rightarrow \\
& \quad \rightarrow|0\rangle\left|\varepsilon\left(\vec{x}_{2}\right)\right\rangle\left|\omega(\vec{k}), \omega\left(\overrightarrow{x_{1}}-\vec{k}-\vec{x}_{2}\right)\right\rangle \rightarrow\left|\Omega_{2}\right\rangle|0\rangle\left|\omega(\vec{k}), \omega\left(\overrightarrow{x_{1}}-\vec{k}-\vec{x}_{2}\right)\right\rangle .
\end{aligned}
$$

Как было показано в $\left[{ }^{6}\right], \tau_{1}$ и $\tau_{2}$ имеют смысл времен пребывания системы в промежуточных состояниях $|0\rangle\left|\varepsilon\left(\vec{\varkappa}_{1}\right)\right\rangle|0\rangle,|0\rangle\left|\varepsilon\left(\vec{\varkappa}_{1}-\vec{k}\right)\right\rangle|\omega(\vec{k})\rangle$ и $|0\rangle\left|\varepsilon\left(\vec{x}_{2}\right)\right\rangle\left|\omega(\vec{k}), \omega\left(\vec{x}_{1}-\vec{k}-\vec{x}_{2}\right)\right\rangle$ для двух амплитуд. Таким образом, время жизни системы в промежуточных состояниях равно $(2 \delta)^{-1}$.

Для проведения классификации на основе критерия, приведенного в $\left[{ }^{5}\right]$, необходимо знать время фазовой памяти $t_{p h}$, т. е. интервал времен $\tau_{1}$ и $\tau_{2}$, за который система забывает частоту первичного фотона. Как видно из (4), забывание частоты первичного фотона обусловлено конечной дисперсией двух фононов, появляющихся в последнем промежуточном состоянии. Формулы (2) и (3) для $W_{2}$ можно представить в виде

$$
\begin{aligned}
& W_{2}=\frac{\left|T_{\vec{x}_{1} \lambda_{1}} T_{\vec{x}_{2} \lambda_{2}}\right|^{2}}{\left[\varepsilon\left(\overrightarrow{x_{1}}\right)-\Omega_{1}\right]^{2}} \int_{-\infty}^{\infty} d t \int_{0}^{\infty} \int_{0}^{\infty} d t_{1} d t^{\prime}{ }_{1} \mathrm{e}^{-\eta\left(t_{1}+t^{\prime}\right)} \times \\
& \times \sum_{\vec{k}}^{\sum} \frac{\left|V(\vec{k}) V\left(\overrightarrow{x_{1}}-\vec{k}-\vec{x}_{2}\right)\right|^{2}}{\left[\varepsilon\left(x_{1}-\vec{k}\right)+\omega(\vec{k})-\Omega_{1}\right]^{2}+\delta^{2}\left(\overrightarrow{x_{1}}-\vec{k}\right)} \times
\end{aligned}
$$

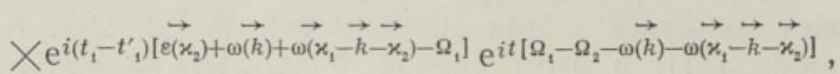

$$
\begin{aligned}
& \eta \rightarrow 0^{+} \text {. }
\end{aligned}
$$

Как видно из (7) (см. также $\left.\left[{ }^{6}\right]\right), t_{1}$ и $t_{1}^{\prime}-$ времена пребывания системы в последнем промежуточном состоянии $|0\rangle\left|\varepsilon\left(\vec{\varkappa}_{2}\right)\right\rangle X$ $\times\left|\omega(\vec{k}), \omega\left(\vec{x}_{1}-\vec{k}-\vec{x}_{2}\right)\right\rangle$ для двух амплитуд. Следовательно, $t_{1}-t^{\prime}{ }_{1}$ характеризует разность фаз в этом состоянии, а интервал $t_{1}-t^{\prime}{ }_{1}$, в котором сумма по $\vec{k}$ в (7) существенно отличается от нуля, - время фазовой памяти. Учитывая, что наиболее сильно от $\vec{k}$ зависит лоренциан под знаком суммы, получим из (7) $t_{p h}=\Delta^{-1}$. Здесь $\Delta-$ суммарная дисперсия фононов $\omega(\vec{k})$ и $\omega\left(\overrightarrow{x_{1}}-\vec{k}-\vec{\varkappa}_{2}\right)$, волновой вектор которых удовлетворяет условию (6).

Если $\Delta^{-1}=t_{p h} \ll(2 \delta)^{-1}$, то за время пребывания в промежуточных состояниях система успевает забыть частоту первичного фотона, и бо́льшую часть рассматриваемого спектра РВС следует, согласно критерию работ $\left[{ }^{5}\right]$, отнести к ГЛ. Выполнению этого условия способствует анизотропия экситонной и фононной зон. Если же $\Delta^{-1}=t_{p h} \geqslant(2 \delta)^{-1}$, получаем либо РКР, либо свечение промежуточного типа. 
Из (4) видно, что ширина спектра $W_{2}$ равна $\Delta$, так что полученный результат согласуется с результатом $\left[{ }^{3}\right]$. работы.

Автор признателен В. В. Хижнякову за обсуждение результатов

\title{
Л И Т Е Р А Т У Р А
}

1. Kle in, M. V. Equivalence of resonance Raman scattering in solids with absorption followed by luminescence. - Phys. Rev. B, 1973, v. 8, N 2, p. 919-921.

2. Permogorov, S. Hot excitons in semiconductors. - Phys. stat. sol. (b), 1975, v. $68, \mathrm{~N} \mathrm{1,} \mathrm{p.} 9-42$.

3. Hizhnyakov, V. V., Sherman, A. V. On the theory of resonant secondary radiation of the excitons weakly interacting with phonons. - Phys. stat. sol. (b), 1978 , v. $85, \mathrm{~N} 1$, p. $51-61$.

4. Hizhnyakov, V., Tehver, I. On the theory of hot luminescence and resonant Raman effect of impurity centres. - Phys. stat. sol., 1970, v. 39, N 1, p. 67-78.

5. Т ехвер И. Ю., Хи жняков В. В. Безызлучательная передача электронного возбуждения в ходе колебательной релаксации. - ЖЭТФ, 1975 , т. 69 , вып. 2 (8), с. $599-610$;

Rebane, K. K., Tehver, I. Y., Hizhnyakov, V. V. On the theory of resonant secondary radiation: scattering, luminescence, and hot luminescence. In: Proc. 1st USA-USSR Symp., New York-London, 1976, p. 393-408.

6. Хи жняков В., Ребане И. Теория переходных спектров резонансного вторичного свечения примесных центров кристаллов. - Изв. АН ЭССР, Физ. Матем., 1977 , т. 26 , № 3 , с. $260-280$.

7. Ивченко Е. Л., Л нг И. Г., П авлов С. Т. Теория резонансного вторичного свечения полупроводников. - ФТТ, 1977, т. 19, № 9, с. $1751-1759$.

\author{
Ннститут физики \\ Академии наук Эстонской ССР
}

Поступила в редакцию 28/II 1978

\section{A. SERMAN}

\section{EKSTSITONIDE RESONANTS-SEKUNDAARKIIRGUSE KLASSIFIKATSIOONIST}

Ajakriteeriumi alusel $\left[{ }^{5}\right]$ on klassifitseeritud Frenkeli ekstsitonide resonants-sekundaarkiirguse spektreid kahe optilise foononi tekkimise korral nulltemperatuuril. On näidatud, et juhul, kui kiirgusspektri laius ületab tunduvalt , ekstsitonide kustumislaiuse, saame kuuma luminestsentsi spektri. Vastupidisel juhul on tegemist kas hajumise või vahepealset tüüpi kiirgusega. Tulemus on täielikus kooskõlas järeldustega, mis on saadud spektraalkriteeriumi alusel $\left[{ }^{3}\right]$.

\section{A. SHERMAN}

\section{ON THE CLASSIFICATION OF RESONANT SECONDARY RADIATION OF EXCITONS}

Resonant secondary radiation spectra of the Frenkel excitons with the generation of two optical phonons at zero temperature are classified on the basis of time criterion [5]. It is shown that in case the spectral width well exceeds that of the exciton decay, the spectrum must be regarded as belonging to hot luminescence. In an opposite case, either scattering or a radiation of an intermediate type is obtained. This inference fully coincides with the one made on the basis of spectral criterion [ $\left.{ }^{3}\right]$. 